\title{
Do cannabis and urbanicity co-participate in causing psychosis? Evidence from a 10-year follow-up cohort study
}

\author{
R. Kuepper ${ }^{1}$, J. van Os ${ }^{1,2 *}$, R. Lieb ${ }^{3,4}$, H.-U. Wittchen ${ }^{3,5}$ and C. Henquet ${ }^{1,6}$ \\ ${ }^{1}$ Department of Psychiatry and Neuropsychology, South Limburg Mental Health Research and Teaching Network, EURON, \\ Maastricht University Medical Center, Maastricht, The Netherlands \\ ${ }^{2}$ King's College London, King's Health Partners, Department of Psychosis Studies, Institute of Psychiatry, London, UK \\ ${ }^{3}$ Max Planck Institute of Psychiatry, Munich, Germany \\ ${ }^{4}$ University of Basel, Department of Psychology, Division of Epidemiology and Health Psychology, Basel, Switzerland \\ ${ }^{5}$ Institute of Clinical Psychology and Psychotherapy, Technical University, Dresden, Germany \\ ${ }^{6}$ Mondriaan Zorggroep, Division Addiction Care, South Limburg, The Netherlands
}

Background. Cannabis use is considered a component cause of psychotic illness, interacting with genetic and other environmental risk factors. Little is known, however, about these putative interactions. The present study investigated whether an urban environment plays a role in moderating the effects of adolescent cannabis use on psychosis risk.

Method. Prospective data ( $n=1923$, aged 14-24 years at baseline) from the longitudinal population-based German Early Developmental Stages of Psychopathology cohort study were analysed. Urbanicity was assessed at baseline and defined as living in the city of Munich (1562 persons per $\mathrm{km}^{2} ; 4061$ individuals per square mile) or in the rural surroundings ( 213 persons per $\mathrm{km}^{2} ; 553$ individuals per square mile). Cannabis use and psychotic symptoms were assessed three times over a 10 -year follow-up period using the Munich version of the Composite International Diagnostic Interview.

Results. Analyses revealed a significant interaction between cannabis and urbanicity $[10.9 \%$ adjusted difference in risk, 95\% confidence interval (CI) 3.2-18.6, $p=0.005$ ]. The effect of cannabis use on follow-up incident psychotic symptoms was much stronger in individuals who grew up in an urban environment (adjusted risk difference $6.8 \%$, $95 \%$ CI 1.0-12.5, $p=0.021$ ) compared with individuals from rural surroundings (adjusted risk difference $-4.1 \%, 95 \%$ CI -9.8 to $1.6, p=0.159$ ). The statistical interaction was compatible with substantial underlying biological synergism.

Conclusions. Exposure to environmental influences associated with urban upbringing may increase vulnerability to the psychotomimetic effects of cannabis use later in life.

Received 9 November 2010; Revised 9 March 2011; Accepted 9 March 2011

Key words: Cannabis, cohort study, interaction, psychosis, urban environment.

\section{Introduction}

Adolescent cannabis use may increase the risk of psychotic disorder (Henquet et al. 2005b; Semple et al. 2005; Moore et al. 2007), the first stage of which is expressed as abnormal persistence of subclinical psychotic symptoms (Cougnard et al. 2007; Kuepper et al. 2011). However, only a minority of cannabis users will eventually develop a psychotic disorder and many individuals with psychotic illness have never been

* Address for correspondence: J. van Os, Ph.D., Department of Psychiatry and Neuropsychology, Maastricht University, PO Box 616 (location DOT12), 6200 MD Maastricht, The Netherlands.

(Email : j.vanos@sp.unimaas.nl) exposed to cannabis. Therefore, cannabis use probably constitutes a component cause, co-depending on other causal influences in shaping risk for psychosis (Henquet et al. 2008). Little is known, however, about the nature of these putative interactions (Caspi et al. 2005; Henquet et al. 2005a; van Os et al. 2010).

Using data from the prospective German Early Developmental Stages of Psychopathology (EDSP) study (Wittchen et al. 1998b; Lieb et al. 2000), Henquet et al. (2005b) showed that individuals scoring high on schizotypy had a much higher risk of developing psychotic symptoms after cannabis use compared with individuals with low or average schizotypy scores (Henquet et al. 2005a). A subsequent study suggested that the moderating effect of schizotypy on the 
psychotomimetic effect of cannabis use may be mediated by genetic risk for psychotic disorder [Genetic Risk and Outcome in Psychosis (GROUP) Investigators, 2011], with some evidence for specific molecular genetic variation (Caspi et al. 2005; Henquet et al. 2006; van Winkel, 2011). Environmental factors may similarly moderate the long-term effects of cannabis on psychosis outcomes, as both Houston et al. (2008) and Harley et al. (2009) reported that individuals who were exposed to trauma early in life, compared with those without trauma, had a much higher risk to develop psychotic outcomes following adolescent cannabis use. Thus, the risk conveyed by the combination of cannabis and trauma was much higher than the sum of the risk posed by either factor alone (Houston et al. 2008; Harley et al. 2009).

Other environmental risk factors may also interact with cannabis use. For example, there is evidence that cannabis use, childhood trauma and growing up in an urban environment independently increase the risk of onset and persistence of psychotic symptoms (Cougnard et al. 2007). It has been hypothesized that all three risk factors may be associated with the same underlying mechanism of behavioural 'sensitization' over time (Collip et al. 2008; van Winkel et al. 2008; Lardinois et al. 2011). Meta-analyses suggest that growing up in an urban environment is consistently associated, in a dose-response fashion, with increased psychosis risk (Krabbendam \& van Os, 2005; March et al. 2008; Kelly et al. 2010), particularly if there is additional evidence of genetic risk (van Os et al. 2003, 2004; Weiser et al. 2007). The aim of the present study was to investigate whether environmental factors associated with urbanicity moderate the strength of the cannabis-psychosis relationship. Data from the EDSP study (Wittchen et al. 1998b; Lieb et al. 2000) were analysed to investigate interaction between cannabis use and urbanicity on later expression of psychotic symptoms in adolescents and young adults.

\section{Method}

\section{Sample and study design}

The EDSP study collected data on the prevalence, incidence, risk factors, co-morbidity and course of mental disorders in a random, representative population sample of adolescents and young adults in the general population (Wittchen et al. 1998b; Lieb et al. 2000). The baseline sample, following ethics committee approval, was randomly drawn, in 1994, from the respective population registry offices of Munich and its 29 counties, to mirror the distribution of individuals expected to be 14-24 years of age at the time of the baseline (T0) interview in 1995. The members of the base population were all those born between 1 June 1970 and 31 May 1981, registered as residents in these localities and having German citizenship. These registers can be regarded as highly accurate because: (1) each German is registered by his town; (2) they are regularly updated; (3) in the interest of scientific studies, any number of randomly drawn addresses with a given sex and age group can be obtained; (4) and strict enforcement of registration by law and the police applies. More details on the sampling, representativeness, instruments, procedures and statistical methods of the EDSP study sample have been presented previously (Wittchen et al. 1998b; Lieb et al. 2000).

The overall design of the cohort study is longitudinal and prospective, consisting of a baseline survey (T0) and three follow-up surveys (T1, T2 and T3), covering time periods on average for T0-T1 of 1.6 (S.D. $=0.2$ ) years, for T0-T2 of 3.5 (S.D. $=0.3$ ) years and for T0-T3 of 8.4 (range $=7.3-10.5$, S.D. $=0.7$ ) years. Because the primary goal of the study was to examine the incidence and developmental risk factors for psychopathology, the younger group (14-15 years), presumed to have the highest incidence density, was sampled at twice the rate of persons aged 16-21 years, and the oldest group (22-24 years) was sampled at half this rate. For the same reason, subjects aged 14-17 years at baseline were examined at the four time points and subjects aged 18-24 years were assessed only three times (T0, T2, T3). The present study is based on the whole cohort assessed at T0, T2 and T3. Response rates were $84 \%(n=2548)$ for $\mathrm{T} 2$ and $74 \%$ $(n=2210)$ for T3.

\section{Instruments}

Participants were assessed using the computerized version of the Munich-Composite International Diagnostic Interview (DIA-X/M-CIDI) (Wittchen et al. 1998a), an updated version of the World Health Organization's Composite International Diagnostic Interview (CIDI) version 1.2 (Robins et al. 1988). The DIA-X/M-CIDI is a comprehensive, fully standardized diagnostic interview and assesses symptoms, syndromes and diagnoses of various mental disorders in accordance with the definitions and criteria of the International Classification of Diseases, Tenth Revision (ICD-10), and the Diagnostic and Statistical Manual of Mental Disorders, Fourth Edition (DSMIV), along with information about onset, duration, severity of symptoms and psychosocial impairment. The CIDI has been primarily designed for use in epidemiological studies of mental disorders and can also be used for clinical purposes. It is divided into $16 \mathrm{sec}-$ tions: one sociodemographic section, 12 sections 
assessing 288 symptoms of groups of mental disorders (including 'somatoform and dissociative', 'phobic and other anxiety', 'depressive and dysthymic', 'manic and bipolar affective', 'schizophrenia and other psychotic', 'eating', 'dementia and other cognitive', 'post-traumatic stress' as well as 'tobacco', 'alcohol' and 'substance-related' disorders) and three final sections containing concluding questions, interviewer observations and interviewer ratings. The instrument, designed for use by trained interviewers who are not clinicians, has shown high inter-rater (Cottler et al. 1991; Wittchen et al. 1991) and test-retest reliability (Wittchen, 1994; Wittchen et al. 1998a). However, the assessment of psychosis with CIDI by lay interviewers is not considered reliable. Therefore, in the EDSP study, trained and experienced clinical interviewers at the level of clinical psychologist, who were allowed to probe with follow-up clinical questions, conducted the interviews in the respondents' homes. At baseline, the DIA-X/M-CIDI lifetime version was used. At each of the follow-up assessments, participants applied the interval version, which covers the period of assessment from the last interview until the next. Data on the G-section concerning psychosis and its clinical relevance were collected at T2 (lifetime version) and T3 (interval version).

\section{Assessment of psychotic experiences}

Data on positive psychotic experiences were collected at time T2 (lifetime version) and T3 (interval version) using the G-section of the DIA-X/M-CIDI. Presence of positive psychotic experiences was broadly defined as any rating of 'present' on any of the 20 DIA-X/ M-CIDI core psychosis items (G1, G2a, G3-G5, G7-G13, G13b, G14, G17, G18, G20, G20C, G21 and G22a), including 14 delusion items, five hallucination items and one item on passivity phenomena. Items relating to classic psychotic symptoms involve, for example, persecution, thought interference, auditory hallucinations and passivity phenomena. Participants were first invited by the psychologist to read a list of all the psychotic experiences and then asked whether they ever experienced such symptoms. All these psychosis items were rated in two ways: 'absent' and 'present'. Thus, all the psychosis DIA-X/M-CIDI items used in the present study were coded in a dichotomous manner without intermediate levels.

\section{Assessment of urbanicity}

Urbanicity was assessed at baseline (T0) and was defined dichotomously as either living in the city of Munich (1562 persons per $\mathrm{km}^{2} ; 4061$ persons per square mile) or in the rural surroundings (213 persons per $\mathrm{km}^{2}$; 553 persons per square mile) at the time of inclusion. Data on living location were derived from the population registry offices of the city and the 29 counties of Munich.

\section{Assessment of cannabis use}

Cannabis use was assessed with the L-section of the DIA-X/M-CIDI at all three assessments. Conforming with previous analyses (Henquet et al. 2005a), cannabis use at T0 was defined as lifetime use of cannabis of five times or more ('T0 cannabis use') and cannabis use at T2 was defined as use of cannabis of five times or more since T0 ('T2 cannabis use').

\section{Statistical analyses}

Data were analysed using Stata, release 10.0 (StataCorp LP, USA). Associations are expressed as risk differences (RDs) from logistic regression models.

\section{Cannabis $\times$ urbanicity interaction}

To investigate whether the effects of incident cannabis use over the T0-T2 period on expression of incident psychotic experiences over the T2-T3 period were moderated by the urban environment, the interaction between T0-T2 cannabis use and urbanicity was calculated under an additive model (van Os et al. 2003, 2004). This was done as the additive interaction can be interpreted in a meaningful way, yielding information on the extent to which two causes biologically depend on each other or co-participate in disease causation (Darroch, 1997). Thus, we derived from the additive statistical interaction an estimate of the amount of biological synergism, as originally described by Darroch (1997), and since successfully applied to psychiatric research (Corcoran \& Malaspina, 2001; van Os et al. 2002; Clarke et al. 2009). The amount of biological synergism in this context can be understood as the proportion of individuals exposed to both cannabis use and urbanicity that developed the psychosis outcome because of the specific co-participation of these two factors. If this proportion is low, the statistical interaction is of little practical importance as most individuals exposed to both cannabis use and urbanicity will have developed the outcome because of either cannabis or urbanicity in isolation, not because of their synergistic action (Darroch, 1997). How the amount of biological synergism can be estimated approximately from the additive statistical interaction has been described in detail previously (Darroch, 1997; van Os et al. 2002).

To ensure prediction of strictly incident psychotic symptoms over the T2-T3 follow-up period, all individuals who had reported lifetime psychotic experiences at T2 were excluded from the analyses. The 


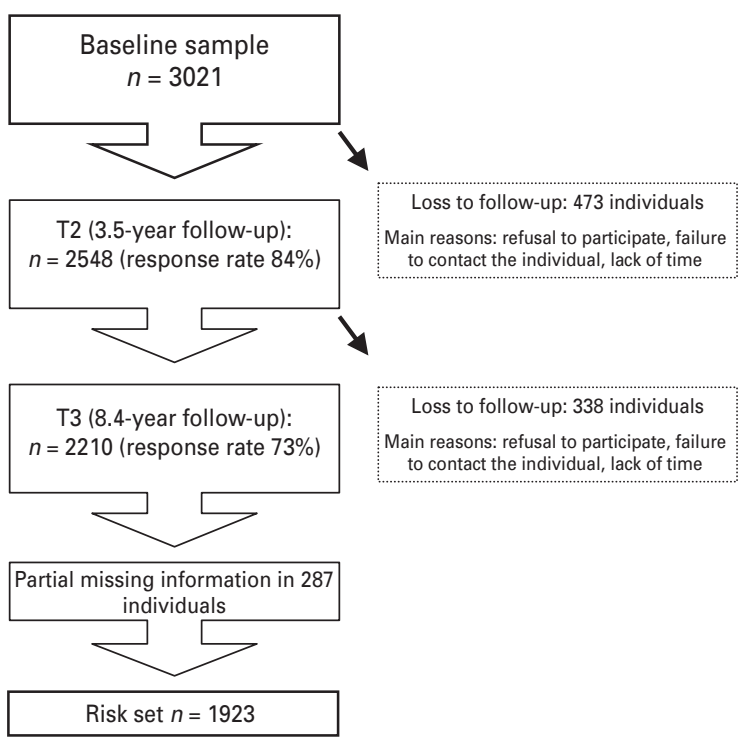

Fig. 1. Schematic illustration of attrition at each stage of the study.

interaction between T0-T2 cannabis use and urbanicity was calculated using the BINREG procedure in Stata yielding RDs, followed by calculation of the appropriate linear combinations from the model with the interaction, using the Stata LINCOM command.

In addition, in order to check for possible mediation between cannabis and urbanicity (in the sense of one factor influencing the occurrence of the other), logistic regression analysis was used to test whether urbanicity was associated with $\mathrm{T} 0$ cannabis use.

All analyses were a priori adjusted for the following confounding risk factors: age (in years); sex $(0=$ female, $1=$ male); socio-economic status (lower, middle, upper, other); cannabis use at baseline (to control for possible mediating effects between urbanicity and cannabis use); use of other drugs; and childhood trauma (Read et al. 2005). Use of 'other drugs' included psychostimulants, sedatives, opiates, cocaine, phencyclidine and psychedelic drugs and refers to use of five times or more of any of these drugs, as assessed with the L-section of the M-CIDI. Conforming to previous analyses in this sample (Spauwen et al. 2006), 'childhood trauma' was defined as lifetime experience of any traumatic experience during childhood as assessed with the $\mathrm{N}$-section of the M-CIDI, dichotomously defined as 'present' versus 'absent'.

\section{Results \\ Sample}

A total of 2210 individuals completed the T3 assessment (response rate $74.0 \%$ ) Of them, 287 participants had partial missing information on substance use and
Table 1. Participant characteristics of the risk set $(n=1923)$

\begin{tabular}{|c|c|c|}
\hline & $n$ & $\%$ \\
\hline \multicolumn{3}{|l|}{ Sex } \\
\hline Male & 926 & 48.2 \\
\hline Female & 997 & 51.8 \\
\hline \multicolumn{3}{|c|}{ Socio-economic status ${ }^{a}$} \\
\hline Lower & 115 & 6.0 \\
\hline Middle & 1114 & 58.0 \\
\hline Upper & 661 & 34.4 \\
\hline Other & 33 & 1.7 \\
\hline \multicolumn{3}{|c|}{ Urbanicity $^{\mathrm{b}}$} \\
\hline Urban & 1345 & 69.9 \\
\hline Rural & 578 & 30.1 \\
\hline \multicolumn{3}{|c|}{ Childhood trauma $^{c}$} \\
\hline Yes & 363 & 18.9 \\
\hline No & 1560 & 81.1 \\
\hline \multicolumn{3}{|c|}{ Cannabis use at $\mathrm{T}^{\mathrm{d}}$} \\
\hline Yes & 247 & 12.8 \\
\hline No & 1676 & 87.2 \\
\hline \multicolumn{3}{|c|}{ Use of other drugs at $\mathrm{T} 0^{\mathrm{d}}$} \\
\hline Yes & 36 & 1.9 \\
\hline No & 1887 & 89.1 \\
\hline
\end{tabular}

T0, Baseline.

a Socio-economic status: lower (lower class, lower middle class); middle (middle middle class); upper (higher middle class, upper class); other (none of the above or missing).

${ }^{\mathrm{b}}$ Urbanicity: urban (the city of Munich: 1562 persons per $\mathrm{km}^{2}$; 4061 persons per square mile); rural (the surroundings of Munich: 213 persons per $\mathrm{km}^{2} ; 553$ persons per square mile).

${ }^{\mathrm{C}}$ Childhood trauma: any traumatic experience during childhood.

${ }^{\mathrm{d}}$ Cannabis use and use of other drugs: any use of more than five times.

psychotic symptoms, resulting in a final risk set for analysis of 1923 individuals of which $926(48.2 \%)$ were men (Fig. 1). Mean age was 18.3 (s.D. $=3.3$ ) years at T0, 21.8 (S.D. =3.4) years at T2 and 26.6 (s.D. =3.5) years at T3. Participant characteristics of the risk set are summarized in Table 1.

At T0, 247 participants (12.8\%) reported lifetime cannabis use. At T2, 392 participants (20.4\%) reported using cannabis over the T0-T2 interval and 381 individuals $(27.1 \%)$ reported $\mathrm{T} 2-\mathrm{T} 3$ interval cannabis use at T3. Psychotic symptoms were reported by 436 participants $(22.7 \%$ - lifetime $)$ at $\mathrm{T} 2$ and by 231 participants $(12.0 \%-$ interval) at T3. At the time of inclusion, 1345 (69.9\%) participants were registered as living in the city of Munich; 578 participants $(30.1 \%)$ were registered as living in the rural surroundings. 
Table 2. Interaction between cannabis use and urbanicity ${ }^{\mathrm{a}}$

\begin{tabular}{|c|c|c|c|c|c|c|c|c|}
\hline & & Number & & & & & $\begin{array}{l}\text { Test for overall } \\
\text { interaction }^{\mathrm{d}}\end{array}$ & \\
\hline $\begin{array}{l}\text { T2 cannabis } \\
\text { use }\end{array}$ & $\begin{array}{l}\text { psychotic } \\
\text { symptoms }^{\text {b }}\end{array}$ & $\begin{array}{l}\text { psychotic } \\
\text { symptoms }^{\mathrm{b}}\end{array}$ & $\begin{array}{l}\text { Psychotic } \\
\text { symptoms, \% }\end{array}$ & $\begin{array}{l}\text { Unadjusted } \\
\text { RD, \% }\end{array}$ & $\begin{array}{l}\text { Adjusted }^{\mathrm{c}} \text { RD } \\
(95 \% \mathrm{CI}), \%\end{array}$ & $p$ & $\begin{array}{l}\text { Adjusted RD } \\
(95 \% \text { CI }), \%\end{array}$ & $p$ \\
\hline \multicolumn{9}{|l|}{ Rural } \\
\hline No & 30 & 355 & 7.8 & \multirow{2}{*}{-2.1} & -4.1 (-9.8 to 1.6$)$ & 0.159 & \multirow{5}{*}{$10.9(3.2-18.6)$} & \multirow{5}{*}{0.005} \\
\hline Yes & 4 & 66 & 5.7 & & & & & \\
\hline Urban & & & & & & & & \\
\hline No & 60 & 774 & 7.2 & \multirow{2}{*}{7.6} & \multirow[t]{2}{*}{6.8 (1.0 to 12.5$)$} & \multirow[t]{2}{*}{0.021} & & \\
\hline Yes & 29 & 167 & 14.8 & & & & & \\
\hline
\end{tabular}

$\mathrm{T} 2$, Second follow-up; RD, risk difference; CI, confidence interval ; T3, third follow-up ; DIA-X/M-CIDI, Munich-Composite International Diagnostic Interview.

${ }^{a}$ Individuals with T2 lifetime psychotic experiences were excluded from the analyses.

${ }^{\mathrm{b}}$ Assessed at T3 as follows: any rating of 'present' on any of the 20 DIA-X/M-CIDI core psychosis items.

${ }^{c}$ Adjusted for age, sex, socio-economic status, baseline cannabis use, use of other drugs and childhood trauma.

d Tests whether cannabis RD in exposure group ('urban') is significantly greater than RD in non-exposure group ('rural').

\section{Main effects of cannabis and urbanicity}

In individuals with no history of psychotic symptoms, cannabis use over the T0-T2 period was significantly associated with incident psychotic symptoms over the T2-T3 period [unadjusted odds ratio $(\mathrm{OR})=1.77,95 \%$ confidence interval (CI) 1.16-2.70, $p=0.008]$. After adjustment for age, sex, socio-economic status, cannabis use at baseline, childhood trauma and use of other drugs, the strength of the association was reduced somewhat (adjusted OR $=1.59$, 95\% CI 0.98-2.60, $p=0.061$ ). There was no significant association between urbanicity and incident psychotic symptoms over the T2-T3 period (adjusted OR $=1.16,95 \% \mathrm{CI}$ $0.77-1.76, p=0.497)$.

\section{Interaction between cannabis and urbanicity}

Analysis revealed a significant interaction between T0-T2 cannabis use and urbanicity (test for additive interaction: $10.9 \%$ adjusted difference in risk, $95 \% \mathrm{CI}$ 3.2-18.6, $p=0.005$; Table 2). The association between T0-T2 cannabis use and T2-T3 psychosis was much stronger for individuals from an urban environment (adjusted RD 6.8\%, 95\% CI 1.0-12.5, $p=0.021$; Table 2) compared with individuals from rural surroundings (adjusted RD $-4.1 \%$, 95\% CI -9.8 to $1.6, p=0.159$; Table 2).

Further analyses revealed that biological synergism was between $51 \%$ and $66 \%$, i.e. of all the individuals in the risk set exposed to both urbanicity and cannabis, the majority had developed the psychosis outcome because of the specific synergistic effect of the two, assuming causality.
There was a small but significant positive association between urbanicity and T0 cannabis use (adjusted OR $=1.4,95 \%$ CI 1.02-1.98, $p=0.038$ ).

\section{Discussion}

There was evidence that urbanicity may moderate the long-term effects of cannabis on psychosis: adolescents who grew up in the city of Munich were much more likely to develop psychotic symptoms after cannabis use than individuals who grew up in the rural surroundings of Munich. This interaction effect was independent of confounding factors such as age, sex, socio-economic status, use of other drugs and childhood trauma and was not irrelevant, as the majority of those exposed to both urbanicity and cannabis developed the psychosis outcome because of their co-action. There was also some evidence that this interaction could be interpreted in the sense of mediation (urbanicity leading to cannabis use leading to psychosis), as a small but significant association existed between urbanicity and cannabis use.

\section{Cannabis and urbanicity: moderation and possible mechanisms}

The present study identified urbanicity as one of the factors that may moderate the association between cannabis and psychosis. Previously, a similar interaction was reported between cannabis and developmental trauma, another environmental exposure associated with psychotic outcomes (Houston et al. 2008; Harley et al. 2009). Given evidence that cannabis, trauma and urbanicity do not reflect the same 
environmental influence in the association with psychotic outcomes (Cougnard et al. 2007), the current analyses, in combination with the findings on similar moderation by developmental trauma (Houston et al. 2008; Harley et al. 2009), suggest that they do share the same interactive pathway. A mechanism of developmental sensitization has been suggested to underlie this pathway (Collip et al. 2008).

Epidemiological evidence indicates that although developmental expression of subclinical psychotic experiences in adolescence is mostly transient, repeated exposure to environmental risk factors causes subclinical psychotic experiences to persist and become more severe, resulting in onset of psychotic illness in a minority of individuals (Cougnard et al. 2007; Dominguez et al. 2011; Mackie et al. 2011). Urbanicity represents a complex proxy environmental influence, and the mechanism of its impact on psychosis remains largely unknown. The effect does not appear to be mediated by demographic factors, obstetric complications, childhood socio-economic position, neuropsychological impairment, air pollution, drug use or ethnic group. However, there is evidence that exposure between the ages of 5 and 15 years is associated with the greatest effect (Krabbendam \& van Os, 2005; March et al. 2008; Kelly et al. 2010), suggesting mediation by factors that have an impact during development. One study showed that an urban environment induced anxiety, negative feelings towards other people and reasoning biases associated with delusional ideation (Ellett et al. 2008). Allardyce et al. (2005) showed that social fragmentation as well as deprivation had a significant effect on first psychosis admission rates independently of urbanicity, and that the effect of urbanicity on first psychosis admission rates disappeared after statistical adjustment for social fragmentation and deprivation (Allardyce et al. 2005). Similarly, there is evidence that risk for psychotic syndrome associated with indicators of social maladjustment, for example single parent family, single marital status and residential instability, similarly varies with the degree to which this represents the exception in relation to the wider social environment (van Os et al. 2000; Zammit et al. 2010). This type of interaction between individual-level and area-level social 'fragmentation' may mediate the effect of the urban environment (Zammit et al. 2010).

The absence of an association between urban environment and incident psychotic symptoms in the current study is in line with previous research (Wiles et al. 2006). The likely explanation is that the time window of exposure to an urban environment in relation to risk for psychosis outcomes is from 5 to 15 years (Pedersen \& Mortensen, 2001). Individuals with lifetime psychotic symptoms, assessed at baseline, that previous work in this sample showed were associated with urban environment (Spauwen et al. 2004), were excluded from the analyses. Lateronset 'incident' psychotic symptoms could not be traced directly anymore to earlier exposure to the urban environment, although an indirect effect was still apparent, through moderation of sensitivity to the psychotomimetic effects of cannabis. It may be hypothesized that early exposure, i.e. between the ages of 5 and 15 years, to increased social fragmentation associated with an urban environment may constitute an environmental stressor that, through epigenetic mechanisms, leads to permanent neurodevelopmental alterations, which in turn may contribute to enhanced sensitivity to the psychotomimetic effects of cannabis later in life.

\section{Cannabis and urbanicity: moderation or mediation?}

In the present study, a small but significant association was found between urbanicity and cannabis use: individuals living in the urban environment were more likely to use cannabis at baseline than individuals from the rural area. This finding indicates that the interaction between cannabis use and urbanicity may represent not only an underlying mechanism of moderation (the psychotomimetic effect of cannabis is larger in urban areas) but also of mediation (living in the city may enhance cannabis use). Both mechanisms would be important from a public health perspective.

\section{Cannabis $\times$ urbanicity: what it does and what it does not tell us?}

One of the limitations of epidemiological research concerns the inability to make inferences about underlying molecular mechanisms. Therefore, our findings are not informative with regard to molecular mechanisms such as developmental sensitization. However, when epidemiological research is followed by targeted experimental animal and human research, it may constitute the first step in a chain of research efforts to uncover the biological mechanisms of environmentenvironment or gene-environment interaction (van Os et al. 2010). Thus, the fact that the majority of those exposed to both cannabis and urbanicity developed the psychosis outcome because of a specific mechanism of co-participation between these two factors suggests that the finding constitutes an important starting point requiring follow-up experimental research targeting a final common pathway that both factors may make an impact on.

The interest of epidemiological findings like these lies in the area of public health and disease causation. As shown by Darroch (1997), evidence of nonadditivity represents a way to estimate the population 
amounts of biological synergism (the population exposed to both risk factors that developed the outcome because of the specific co-participation between the two factors) and biological parallelism (the population exposed to both risk factors that developed the outcome because of the action of only one factor). Examination of biological synergism and parallelism is of vital interest to epidemiologists and public health, since it allows identification of populations that would benefit most from a given intervention: if synergism is large, public health gains could be obtained by targeting either factor. On the other hand, if parallelism is large, public health may gain by targeting both factors. In the current study, synergism was between 50\% and $65 \%$, indicating that targeting either factor could potentially result in public health gains.

\section{Limitations and methodological issues}

There are several limitations. First, information on substance use and psychosis outcome was acquired using the M-CIDI, which is meant to essentially provide self-reported information. Nevertheless, the interview was conducted face-to-face by clinical psychologists who were allowed to follow up with clinical questioning in order to ensure systematic and valid assessment of outcomes. This method can therefore be assumed to yield better and more valid results than a self-report questionnaire.

Second, urbanicity was assessed by retrieving data on living location from the German registry offices. Although these registers can be considered as highly accurate since in Germany registration is obligatory and registries are regularly updated, the information is restricted to current living location and therefore does not provide information on duration of living at a certain location. Pedersen \& Mortensen (2001) showed that increasing duration of living in urban areas leads to increased psychosis risk in a dose-response fashion. Research has furthermore suggested that the effect of urbanicity on psychosis risk operates during upbringing rather than at birth (Pedersen \& Mortensen, 2001) or around illness onset (Marcelis et al. 1999). Considering the current study, even under the hypothetical assumption that participants registered as living in the city of Munich had just moved there from the rural surroundings or, the other way round, individuals from the rural surroundings had just moved to the city, this would have yielded an underestimation, rather than an overestimation of the effect size. In addition, previous work suggests that current urban residence in young people reflects urban upbringing in the majority (Marcelis et al. 1999).

The paradigm used in the current study was based on the notion that psychotic symptoms can be expressed below the threshold of diagnosis and are meaningful in relation to the clinical phenotype. Metaanalytic work of subdiagnostic expression of psychotic experiences in the general population has shown aetiological, psychopathological and longitudinal continuity between the extended subclinical phenotype and clinical expression of psychotic disorder, as well as a similar age-related developmental pattern of expression (van Os et al. 2009); a recent landmark general population birth cohort study confirmed this pattern of findings (Polanczyk et al. 2009). Further, since transient and normally expressed psychotic experiences may, under certain circumstances, become abnormally persistent and develop into clinical psychosis (Hanssen et al. 2005; Cougnard et al. 2007; Dominguez et al. 2011), psychotic experiences represent an important phenotype for the investigation of mechanisms and pathways by which environmental risk factors such as cannabis make an impact on psychosis risk (Kuepper et al. 2011).

Finally, in interpreting the findings of this current study, it was assumed that in this area in Germany, cannabis used in the city does not differ from cannabis used in rural areas. Although this cannot be formally explored the assumption may be valid, since availability of cannabis in Germany is limited in general (compared with countries such as the Netherlands, where 'coffee shops' are allowed to sell cannabis on a semi-legal basis) and access to cannabis is probably comparable between rural and urban areas.

\section{Acknowledgements}

C.H. was supported by the Dutch Medical Research Council (VENI grant). This work is a part of the EDSP study and is funded by the German Federal Ministry of Education and Research (BMBF) project no. 01EB9405/6, 01EB9901/6, EB01016200, 01EB0140 and 01EB0440.

\section{Declaration of Interest}

J.v.O. is/has been an unrestricted research grant holder with, or has received financial compensation as an independent symposium speaker from, Eli Lilly, BMS, Lundbeck, Organon, Janssen-Cilag, GSK, AstraZeneca, Pfizer and Servier, companies that have an interest in the treatment of psychosis.

\section{References}

Allardyce J, Gilmour H, Atkinson J, Rapson T, Bishop J, McCreadie RG (2005). Social fragmentation, deprivation and urbanicity: relation to first-admission rates for psychoses. British Journal of Psychiatry 187, 401-406. 
Caspi A, Moffitt TE, Cannon M, McClay J, Murray R, Harrington H, Taylor A, Arseneault L, Williams B, Braithwaite A, Poulton R, Craig IW (2005). Moderation of the effect of adolescent-onset cannabis use on adult psychosis by a functional polymorphism in the catechol-O-methyltransferase gene: longitudinal evidence of a gene $\mathrm{X}$ environment interaction. Biological Psychiatry 57, 1117-1127.

Clarke MC, Tanskanen A, Huttunen M, Whittaker JC, Cannon M (2009). Evidence for an interaction between familial liability and prenatal exposure to infection in the causation of schizophrenia. American Journal of Psychiatry 166, 1025-1030.

Collip D, Myin-Germeys I, van Os J (2008). Does the concept of 'sensitization' provide a plausible mechanism for the putative link between the environment and schizophrenia? Schizophrenia Bulletin 34, 220-225.

Corcoran C, Malaspina D (2001). Traumatic brain injury and schizophrenia. International Journal of Mental Health 30, 17-33.

Cottler LB, Helzer JE, Mager D, Spitznagel EL, Compton WM (1991). Agreement between DSM-III and III-R substance use disorders. Drug and Alcohol Dependence 29, 17-25.

Cougnard A, Marcelis M, Myin-Germeys I, De Graaf R, Vollebergh W, Krabbendam L, Lieb R, Wittchen HU, Henquet C, Spauwen J, van Os J (2007). Does normal developmental expression of psychosis combine with environmental risk to cause persistence of psychosis? A psychosis proneness-persistence model. Psychological Medicine 37, 513-527.

Darroch J (1997). Biologic synergism and parallelism. American Journal of Epidemiology 145, 661-668.

Dominguez MD, Wichers M, Lieb R, Wittchen HU, van Os J (2011). Evidence that onset of clinical psychosis is an outcome of progressively more persistent subclinical psychotic experiences: an 8-year cohort study. Schizophrenia Bulletin 37, 84-93.

Ellett L, Freeman D, Garety PA (2008). The psychological effect of an urban environment on individuals with persecutory delusions : the Camberwell walk study. Schizophrenia Research 99, 77-84.

Genetic Risk and Outcome in Psychosis (GROUP) Investigators (2011). Evidence that familial liability for psychosis is expressed as differential sensitivity to cannabis: an analysis of patient-sibling and sibling-control pairs. Archives of General Psychiatry 68, 138-147.

Hanssen M, Bak M, Bijl R, Vollebergh W, van Os J (2005). The incidence and outcome of subclinical psychotic experiences in the general population. British Journal of Clinical Psychology 44, 181-191.

Harley M, Kelleher I, Clarke M, Lynch F, Arseneault L, Connor D, Fitzpatrick C, Cannon M (2009). Cannabis use and childhood trauma interact additively to increase the risk of psychotic symptoms in adolescence. Psychological Medicine 40, 1627-1634.

Henquet C, Di Forti M, Morrison P, Kuepper R, Murray RM (2008). Gene-environment interplay between cannabis and psychosis. Schizophrenia Bulletin 34, 1111-1121.
Henquet C, Krabbendam L, Spauwen J, Kaplan C, Lieb R, Wittchen HU, van Os J (2005a). Prospective cohort study of cannabis use, predisposition for psychosis, and psychotic symptoms in young people. British Medical Journal 330, 11.

Henquet C, Murray R, Linszen D, van Os J (2005b). The environment and schizophrenia: the role of cannabis use. Schizophrenia Bulletin 31, 608-612.

Henquet C, Rosa A, Krabbendam L, Papiol S, Fananas L, Drukker M, Ramaekers JG, van Os J (2006). An experimental study of catechol-O-methyltransferase Val ${ }^{158}$ Met moderation of $\Delta$-9-tetrahydrocannabinolinduced effects on psychosis and cognition. Neuropsychopharmacology 31, 2748-2757.

Houston JE, Murphy J, Adamson G, Stringer M, Shevlin M (2008). Childhood sexual abuse, early cannabis use, and psychosis: testing an interaction model based on the National Comorbidity Survey. Schizophrenia Bulletin 34, 580-585.

Kelly BD, O'Callaghan E, Waddington JL, Feeney L, Browne S, Scully PJ, Clarke M, Quinn JF, McTigue O, Morgan MG, Kinsella A, Larkin C (2010). Schizophrenia and the city: a review of literature and prospective study of psychosis and urbanicity in Ireland. Schizophrenia Research 116, 75-89.

Krabbendam L, van Os J (2005). Schizophrenia and urbanicity: a major environmental influence-conditional on genetic risk. Schizophrenia Bulletin 31, 795-799.

Kuepper R, van Os J, Lieb R, Wittchen HU, Hofler M, Henquet C (2011). Continued cannabis use and risk of incidence and persistence of psychotic symptoms: 10 year follow-up cohort study. British Medical Journal 342, $\mathrm{d} 738$

Lardinois M, Lataster T, Mengelers R, van Os J, MyinGermeys I (2011). Childhood trauma and increased stress sensitivity in psychosis. Acta Psychiatrica Scandinavica 123, 28-35.

Lieb R, Isensee B, von Sydow K, Wittchen HU (2000). The Early Developmental Stages of Psychopathology Study (EDSP): a methodological update. European Addiction Research 6, 170-182.

Mackie CJ, Castellanos-Ryan N, Conrod PJ (2011). Developmental trajectories of psychotic-like experiences across adolescence: impact of victimization and substance use. Psychological Medicine 41, 47-58.

Marcelis M, Takei N, van Os J (1999). Urbanization and risk for schizophrenia: does the effect operate before or around the time of illness onset? Psychological Medicine 29, 1197-1203.

March D, Hatch SL, Morgan C, Kirkbride JB, Bresnahan M, Fearon P, Susser E (2008). Psychosis and place. Epidemiologic Reviews 30, 84-100.

Moore TH, Zammit S, Lingford-Hughes A, Barnes TR, Jones PB, Burke M, Lewis G (2007). Cannabis use and risk of psychotic or affective mental health outcomes: a systematic review. Lancet 370, 319-328.

Pedersen CB, Mortensen PB (2001). Evidence of a dose-response relationship between urbanicity during upbringing and schizophrenia risk. Archives of General Psychiatry 58, 1039-1046. 
Polanczyk G, Moffitt TE, Arseneault L, Cannon M, Ambler A, Keefe RS, Houts R, Odgers CL, Caspi A (2010). Etiological and clinical features of childhood psychotic symptoms: results from a birth cohort. Archives of General Psychiatry 67, 328-338.

Read J, van Os J, Morrison AP, Ross CA (2005). Childhood trauma, psychosis and schizophrenia: a literature review with theoretical and clinical implications. Acta Psychiatrica Scandinavica 112, 330-350.

Robins LN, Wing J, Wittchen HU, Helzer JE, Babor TF, Burke J, Farmer A, Jablenski A, Pickens R, Regier DA, Sartorius N, Towle LH (1988). The Composite International Diagnostic Interview. An epidemiologic instrument suitable for use in conjunction with different diagnostic systems and in different cultures. Archives of General Psychiatry 45, 1069-1077.

Semple DM, McIntosh AM, Lawrie SM (2005). Cannabis as a risk factor for psychosis: systematic review. Journal of Psychopharmacology 19, 187-194.

Spauwen J, Krabbendam L, Lieb R, Wittchen HU, van Os J (2004). Does urbanicity shift the population expression of psychosis? Journal of Psychiatric Research 38, 613-618.

Spauwen J, Krabbendam L, Lieb R, Wittchen HU, van Os J (2006). Evidence that the outcome of developmental expression of psychosis is worse for adolescents growing up in an urban environment. Psychological Medicine 36, 407-415.

van Os J, Bak M, Hanssen M, Bij1 RV, de Graaf R, Verdoux H (2002). Cannabis use and psychosis: a longitudinal population-based study. American Journal of Epidemiology 156, 319-327.

van Os J, Driessen G, Gunther N, Delespaul P (2000). Neighbourhood variation in incidence of schizophrenia. Evidence for person-environment interaction. British Journal of Psychiatry 176, 243-248.

van Os J, Hanssen M, Bak M, Bijl RV, Vollebergh W (2003). Do urbanicity and familial liability coparticipate in causing psychosis? American Journal of Psychiatry 160, 477-482.

van Os J, Kenis G, Rutten BP (2010). The environment and schizophrenia. Nature 468, 203-212.

van Os J, Linscott RJ, Myin-Germeys I, Delespaul P, Krabbendam L (2009). A systematic review and meta-analysis of the psychosis continuum: evidence for a psychosis proneness-persistence-impairment model of psychotic disorder. Psychological Medicine 39, 179-195. van Os J, Pedersen CB, Mortensen PB (2004). Confirmation of synergy between urbanicity and familial liability in the causation of psychosis. American Journal of Psychiatry 161, 2312-2314.

van Winkel R; Genetic Risk and Outcome in Psychosis (GROUP) Investigators (2011). Family-based analysis of genetic variation underlying psychosis-inducing effects of cannabis: sibling analysis and proband follow-up. Archives of General Psychiatry 68, 148-157.

van Winkel R, Stefanis NC, Myin-Germeys I (2008). Psychosocial stress and psychosis. A review of the neurobiological mechanisms and the evidence for genestress interaction. Schizophrenia Bulletin 34, 1095-1105.

Weiser M, van Os J, Reichenberg A, Rabinowitz J, Nahon D, Kravitz E, Lubin G, Shmushkevitz M, Knobler HY, Noy S, Davidson M (2007). Social and cognitive functioning, urbanicity and risk for schizophrenia. British Journal of Psychiatry 191, 320-324.

Wiles NJ, Zammit S, Bebbington P, Singleton N, Meltzer H, Lewis G (2006). Self-reported psychotic symptoms in the general population: results from the longitudinal study of the British National Psychiatric Morbidity Survey. British Journal of Psychiatry 188, 519-526.

Wittchen HU (1994). Reliability and validity studies of the WHO-Composite International Diagnostic Interview (CIDI) : a critical review. Journal of Psychiatry Research 28, 57-84.

Wittchen HU, Lachner G, Wunderlich U, Pfister H (1998a). Test-retest reliability of the computerized DSM-IV version of the Munich-Composite International Diagnostic Interview (M-CIDI). Social Psychiatry and Psychiatric Epidemiology 33, 568-578.

Wittchen HU, Perkonigg A, Lachner G, Nelson CB (1998b). Early developmental stages of psychopathology study (EDSP): objectives and design. European Addiction Research 4, 18-27.

Wittchen HU, Robins LN, Cottler LB, Sartorius N, Burke JD, Regier D (1991). Cross-cultural feasibility, reliability and sources of variance of the Composite International Diagnostic Interview (CIDI). The Multicentre WHO/ADAMHA Field Trials. British Journal of Psychiatry 159, 645-653, 658.

Zammit S, Lewis G, Rasbash J, Dalman C, Gustafsson JE, Allebeck P (2010). Individuals, schools, and neighborhood: a multilevel longitudinal study of variation in incidence of psychotic disorders. Archives of General Psychiatry 67, 914-922. 